\title{
Editorial
}

\section{Management of hepatocellular carcinoma}

Received: 21 September 2021; Accepted: 6 October 2021;

Published: 08 October 2021.

*Correspondence: Salem Youssef Mohamed, salemyousefmohamed@gmail.com

ORCID: https://orcid.org/o0oo-0003-2917-4293

DOI: https://doi.org/10.52378/mhae1978

Cell phone: +201147805292.

Hepatocellular carcinoma ( $\mathrm{HCC}$ ) is a heterogeneous disease that develops most of the time in the diseased liver. Early diagnosis and management are the best options for patients, so screening programs are mandatory for those at risk of developing HCC. Despite the challenges of management of HCC, there is hopeful management for HCC as the field of primary liver cancer has moved quickly.

Keywords: hepatocellular carcinoma, management, epidemiology, immune checkpoint inhibitors.

1-New trends in epidemiology and surveillance of HCC: virally induced tumors will soon become less frequent, alcohol-related cirrhosis is a stably prevalent pre-neoplastic condition, fatty liver disease is a growing cause of HCC even in the absence of cirrhosis[1].

2- Pathogenesis: Recent technological breakthroughs such as next-generation sequencing have enabled the sequencing of a whole tumor exome, genome, or transcriptome in a few hours. Sequencing data have highlighted the main signaling pathways altered in HCC (e.g., Telomere, cell cycle, epigenetic)[1]. These data helped understand the link between genetic alterations clinical and pathological features of HCC[2], [3]. Telomerase activation, viral insertions, chromosome translocation, or gene amplification are the most frequent driver gene alterations in $80 \%$ of $\mathrm{HCC}[4]$.

\section{3- Trends in Diagnosis:}

The fraction of AFP bound to lectin [5], des-gamma-carboxyprothrombin[5], Golgi protein-73 [6], glypican-3 [7], or Dickkopf-1[8] present the same drawbacks as the AFP and cannot compete with the reliability of the ultrasonography.

Dynamic imaging study (CT or MRI): The difference between 'dynamic' and 'time-lapse' can be considered a smooth motion picture and a stop motion animation. The advantage of performing dynamic CT is achieving uninterrupted, real in situ experiments.

The use of contrast ultrasound has been proposed as a second-rate test in the latest update of the EASL[9] guidelines. 
$\underline{\text { PET }}$ is of poor performance for diagnosing HCC. ${ }^{11} \mathrm{C}$-Choline shows promising initial results, but not comparable with CT or MRI[10].

4-Resection of HCC and liver transplantation: the times when cirrhosis was considered an absolute contraindication for liver resection are over. Vibert et al. summarize the recent advances in the surgical treatment of HCC.[11] Lower morbidity rates associated with the laparoscopic approaches, and personalized prognostication based on the volume and quality of the future liver remnant, the degree of portal hypertension, and the risk of tumor recurrence have changed the way surgery is considered in the treatment of HCC[12].

Adjuvant or neoadjuvant treatment: Tumor recurrence complicates $70 \%$ of cases 5 years after surgical resection. Checkpoint inhibitors(ICls) could reduce recurrence and increase early survival after curative treatment[13]. Many programs propose using AFP levels and response Neoadjuvant treatment ( downstaging ) as markers of good biological behavior to be included in the selection criteria [14]. The "compound criteria" that, in addition to the size and number of nodules, provide information on tumor biology (e.g., AFP) and include tumor evolution and response to previous treatments, could replace the criteria in the future.

5-Locoregional treatment: Palmer et al. summarize the significant issues of TACE, including risk stratification for patient selection, the transition from TACE to systemic therapy, and the use of TACE or other locoregional medicines (e.g., TARE) for patients with the earlier-stage disease (for downsizing)[15]. Data from clinical trials of combinations of TACE and systemic therapy for BCLC stage $\mathrm{B}$ or $\mathrm{C}$ patients are under evaluation [16].

Ablation techniques as laser[17], cryoablation[18], high-intensity focused ultrasound[19], or irreversible electroporation[20] are not superior to RFA.

Radiomics: CT-guided electromagnetic navigation systems and image fusion are available, allowing refinement of percutaneous ablation. They provide precise and safer punctures, reduce the operator-dependent factor, and allow access to hard-to-reach locations.

The safety and efficacy of synthetic microspheres loaded with adriamycin [ drug-eluting bead] TACE) are promising. The charged particles achieve vascular occlusion with a slow release of chemotherapy at the intratumoral level. This allows less passage of chemotherapy to the systemic circulation and thus reduces the potential toxicity of chemotherapy once the particles are injected[21]. The immunotherapy combination with ablation has been suggested to increase survival for several years[22].

Radiation therapy techniques such as three-dimensional conformal radiation therapy, intensitymodulated radiation therapy, image-guided stereotaxic radiation therapy, or proton beam radiation allow high doses of radiation to the tumor without damaging the surrounding tissue. The results reported in HCC and recurrent $\mathrm{HCC}$ are promising, but efficacy needs to be confirmed [23],[24]. 
6-Assessment of radiological response: Modified Response Evaluation Criteria in Solid Tumors has served its purpose since being included in clinical practice guidelines[25] for the Management of HCC. Using Al techniques to aid traditional diagnostic procedures is promising (diagnosis, evaluation of recurrence, survival)[26].

7- Selection of cells expressing the cell surface epithelial cell adhesion molecule (EpCAM): used for circulating tumor cells (CTC) enrichment as it has little or no expression on leukocytes and is expressed by most epithelial-derived cancers. The FDA-cleared Cell Search platform uses CTC enrichment by EpCAM targeting immunomagnetic selection. Liquid biopsy is a procedure based on identifying tumor components released into biological fluids, particularly blood. CTC, circulating tumor nucleic acids, DNA and RNA, and extracellular vesicles[27].

8-Immunotherapy (immune checkpoint inhibitors(ICls) and tyrosine kinase inhibitors(TKIs) as mono or combined): TKIs: After a decade of struggling with negative results from randomized trials of drug therapy for advanced HCC, data emerging over the past three years has transformed the management landscape [28]. Faivre et al. provide an overview of targeted therapies for advanced HCC, including Sorafenib and lenvatinib in the first-line setting and regorafenib, cabozantinib, and Ramucirumab in the second-line setting[29]. Sorafenib and lenvatinib improve survival in HCC patients compared to the first-line placebo. Second-line, regorafenib improves survival in patients who progress and are tolerant to Sorafenib, cabozantinib in patients who are candidates for second and third-line treatments, and Ramucirumab in patients who are candidates for second-line treatments who present an AFP value $\geq 400 \mathrm{ng} / \mathrm{dL}$. Ramucirumab is a monoclonal antibody that binds VEGFR-2.

\section{ICls:}

ICls were the first agents, other than tyrosine kinase inhibitors, to be approved for the treatment of HCC[30],[31].

Combinations of ICls targeting the programmed cell death-1 (PD-1) pathway and anti-angiogenic therapy have become the mainstream of combination therapy trials for $\mathrm{HCC}[32],[33,34]$. A combination of atezolizumab and bevacizumab was the first treatment shown to improve the benefit of Sorafenib [35]. Atezolizumab is a PD-L1 inhibitor, and bevacizumab is a monoclonal antibody against VEGF[36].

A combination of nivolumab and ipilimumab (cytotoxic associated lymphocyte-associated protein 4) will be approved soon. Cheng et al. discuss advances in developing predictive biomarkers[37]. In addition, they discuss the further development challenges of different immunotherapy-based combination therapies[37],[38]. Although ICls are well tolerated, they can result in life-threatening toxicities[39]. The management of such toxicities in patients with HCC, who usually suffer from advanced chronic liver disease, is challenging[40], specific recommendations for liver toxicities were formulated [41]. 
Adoptive cellular therapy: A recent review summarizes chimeric antigen receptor $\mathrm{T}$ cell(CAR-T) therapy targets HCC and current obstacles [42].

\section{Footnotes}

Citation of this article: Mohamed SY. Management of hepatocellular carcinoma. African journal of gastroenterology and hepatology [Internet]. Egypt's Presidential Specialized Council for Education and Scientific Research; 2021 Oct 8;4(1):19-25. Available from: http://dx.doi.org/10.52378/mhae1978.

Peer-reviewers: Mohamed Hassan Ali Emara (professor of hepatology, gastroenterology, and infectious diseases), Emad Fawzi Hamed (professor of internal medicine).

\section{E- Editor: Salem Y Mohamed.}

Copyright (C). This open-access article is distributed under the Creative Commons Attribution License (CC BY). The use, distribution, or reproduction in other forums is permitted, provided the original author(s) and the copyright owner(s) are credited. The original publication in this journal is cited by accepted academic practice. No use, distribution, or reproduction is permitted, which does not comply with these terms.

Disclaimer: All claims expressed in this article are solely those of the authors and do not necessarily represent their affiliated organizations or those of the publisher, the editors, and the reviewers. Any product that may be evaluated in this article or claim that its manufacturer may make is not guaranteed or endorsed by the publisher. 


\section{References}

1. Nault, J.C., et al., Milestones in the pathogenesis and management of primary liver cancer. J Hepatol, 2020. 72(2): p. 209-214.

2. Ally, A., et al., Comprehensive and integrative genomic characterization of hepatocellular carcinoma. Cell, 2017. 169(7): p. 1327-1341. e23.

3. Hoshida, Y. et al., Integrative transcriptome analysis reveals common molecular subclasses of human hepatocellular carcinoma. Cancer Research, 2009. 69(18): p. 7385-7392.

4. Schulze, K. et al., Exome sequencing of hepatocellular carcinomas identifies new mutational signatures and potential therapeutic targets. Nature genetics, 2015. 47(5): p. 505-511.

5. $\quad$ Marrero, J.A., et al., $\alpha$-fetoprotein, des- $\gamma$ carboxyprothrombin, and lectin-bound $\alpha$-fetoprotein in early hepatocellular carcinoma. Gastroenterology, 2009. 137(1): p. 110-118.

6. Marrero, J.A., et al., GP73, a resident Golgi glycoprotein, is a novel serum marker for hepatocellular carcinoma. Journal of hepatology, 2005. 43(6): p. 1007-1012.

7. Capurro, M., et al., Glypican-3: a novel serum and histochemical marker for hepatocellular carcinoma. Gastroenterology, 2003. 125(1): p. 89-97.

8. Shen, Q., et al., Serum DKK1 as a protein biomarker for the diagnosis of hepatocellular carcinoma: a large-scale, multicentre study. The lancet oncology, 2012. 13(8): p. 817-826.

9. Liver, E.A.F.T.S.O.T., EASL clinical practice guidelines: Management of hepatocellular carcinoma. Journal of hepatology, 2018. 69(1): p. 182-236.

10. Chotipanich, C., et al., Diagnosis of Hepatocellular Carcinoma Using C11 Choline PET/CT: Comparison with F18 FDG, ContrastEnhanced MRI, and MDCT. Asian Pacific Journal of Cancer Prevention, 2016. 17(7): p. 3569-3573.

11. Vibert, E., M. Schwartz, and K.M. Olthoff, Advances in resection and transplantation for hepatocellular carcinoma. Journal of hepatology, 2020. 72(2): p. 262-276.

12. Chan, A.W., et al., Development of pre and post-operative models to predict early recurrence of hepatocellular carcinoma after surgical resection. Journal of hepatology, 2018. 69(6): p. 12841293.

13. Lee, J.H., et al., Adjuvant immunotherapy with autologous cytokine-induced killer cells for hepatocellular carcinoma. Gastroenterology, 2015. 148(7): p. 1383-1391. e6.

14. Lysy, P.A., et al., Human skin fibroblasts: From mesodermal to hepatocyte-like differentiation. Hepatology, 2007. 46(5): p. 1574-1585.

15. Palmer, D.H., K. Malagari, and L.M. Kulik, Role of locoregional therapies in the wake of systemic therapy. Journal of hepatology, 2020. 72(2): p. 277-287.

16. Meyer, T., et al., Sorafenib in combination with transarterial chemoembolisation in patients with unresectable hepatocellular carcinoma (TACE 2): a randomised placebo-controlled, double-blind, phase 3 trial. The lancet Gastroenterology \& hepatology, 2017. 2(8): p. 565-575.

17. Francica, G., et al., Effectiveness, safety, and local progression after percutaneous laser ablation for hepatocellular carcinoma nodules up to $4 \mathrm{~cm}$ are not affected by tumor location. American Journal of Roentgenology, 2012. 199(6): p. 1393-1401.

18. Wang, C., et al., Multicenter randomized controlled trial of percutaneous cryoablation versus radiofrequency ablation in hepatocellular carcinoma. Hepatology, 2015. 61(5): p. 1579-1590.

19. Ng, K.K., et al., High-intensity focused ultrasound for hepatocellular carcinoma: a single-center experience. Annals of surgery, 2011. 253(5): p. 981-987.

20. Sutter, O., et al., safety and efficacy of irreversible electroporation for the treatment of hepatocellular carcinoma not amenable to thermal ablation techniques: a retrospective singlecenter case series. Radiology, 2017. 284(3): p. 877-886. 
21. Alter, M.J., Epidemiology of viral hepatitis and HIV co-infection. Journal of hepatology, 2006. 44: p. S6-S9.

22. Kudo, M., et al., Orantinib versus placebo combined with transcatheter arterial chemoembolisation in patients with unresectable hepatocellular carcinoma (ORIENTAL): a randomised, double-blind, placebo-controlled, multicentre, phase 3 study. The Lancet Gastroenterology \& Hepatology, 2018. 3(1): p. 37-46.

23. Thomas, H.R. and M. Feng, Stereotactic Body Radiation Therapy (SBRT) in Hepatocellular Carcinoma. Current Hepatology Reports: p. 1-11.

24. Kim, T.H., et al., Proton beam radiotherapy vs. radiofrequency ablation for recurrent hepatocellular carcinoma: A randomized phase III trial. Journal of Hepatology, 2020.

25. Heimbach, J.K., et al., AASLD guidelines for the treatment of hepatocellular carcinoma. Hepatology, 2018. 67(1): p. 358-380.

26. Pérez, M.J. and R.G. Grande, Application of artificial intelligence in the diagnosis and treatment of hepatocellular carcinoma: A review. World Journal of Gastroenterology, 2020. 26(37): p. 5617.

27. Mann, J., H.L. Reeves, and A.E. Feldstein, Liquid biopsy for liver diseases. Gut, 2018. 67(12): p. 2204-2212.

28. Zhu, A.X., et al., Ramucirumab after Sorafenib in patients with advanced hepatocellular carcinoma and increased $\alpha$-fetoprotein concentrations (REACH-2): a randomised, double-blind, placebocontrolled, phase 3 trial. The lancet oncology, 2019. 20(2): p. 282-296.

29. Faivre, S., L. Rimassa, and R.S. Finn, Molecular therapies for HCC: Looking outside the box. Journal of hepatology, 2020. 72(2): p. 342-352.

30. Llovet, J.M., et al., Hepatocellular carcinoma. Nature Reviews Disease Primers, 2021. 7(1): p. 6.

31. Forner, A., et al., Diagnóstico y tratamiento del carcinoma hepatocelular. Actualización del documento de consenso de la AEEH, SEOM, SERAM, SERVEI y SETH. Medicina clinica, 2016. 146(11): p. 511. e1-511. e22.

32. Llovet, J.M., et al., Molecular therapies and precision medicine for hepatocellular carcinoma. Nature reviews Clinical oncology, 2018. 15(10): p. 599-616.

33. Greten, T.F., et al., Targeted and immune-based therapies for hepatocellular carcinoma. Gastroenterology, 2019. 156(2): p. 510-524.

34. El Dika, I., I. Makki, and G.K. Abou-Alfa, Hepatocellular carcinoma, novel therapies on the horizon. Chinese Clinical Oncology, 2020.

35. Cheng, A.-L., et al., IMbrave150: efficacy and safety results from a ph III study evaluating atezolizumab (atezo)+ bevacizumab (bev) vs Sorafenib (Sor) as first treatment (tx) for patients (pts) with unresectable hepatocellular carcinoma (HCC). Annals of Oncology, 2019. 30: p. ix186-ix187.

36. Vogel, A. and A. Saborowski, Current strategies for the treatment of intermediate and advanced hepatocellular carcinoma. Cancer treatment reviews, 2020. 82: p. 101946.

37. Cheng, A.-L., et al., Challenges of combination therapy with immune checkpoint inhibitors for hepatocellular carcinoma. Journal of hepatology, 2020. 72(2): p. 307-319.

38. Greten, T.F., et al., Combined locoregional-immunotherapy for liver cancer. Journal of hepatology, 2019. 70(5): p. 999-1007.

39. Postow, M.A., R. Sidlow, and M.D. Hellmann, Immune-related adverse events associated with immune checkpoint blockade. New England Journal of Medicine, 2018. 378(2): p. 158-168.

40. Ghavimi, S., et al., management and treatment of hepatocellular carcinoma with immunotherapy: a review of current and future options. Journal of Clinical and Translational Hepatology, 2020. 8(2): p. 168.

41. Sangro, B., et al., Diagnosis and Management of toxicities of immune checkpoint inhibitors in hepatocellular carcinoma. Journal of hepatology, 2020. 72(2): p. 320-341. 
42. Guo, J. and Q. Tang, Recent updates on chimeric antigen receptor T cell therapy for hepatocellular carcinoma. Cancer Gene Therapy, 2021. 